\title{
Esnek Algılayıcı Kontrollü Robot EI Tasarımı ve Gerçeklenmesi
}

\author{
${ }^{1 *}$ S. Serdar ÖZKAN, ${ }^{2}$ Durmuş Karayel, ${ }^{3}$ Gökhan ATALI, ${ }^{4}$ İbrahim GÖKBAYRAK \\ ${ }^{1,2,3}$ Sakarya Üniversitesi, Teknoloji Fakültesi Mekatronik Mühendisliği
}

\section{$\ddot{O ̈ z}$}

Dünya genelinde artan yalın üretim sistemlerinin gelişmesinde şüphesiz en önemli değere sahip olan girdilerden birisi de robotik çalışmalardır. Robotik çalışmalar sayesinde üretimde oluşabilecek hata payları ve kaza oranları en aza indirilerek üretimler gerçekleştirilebilmektedir. Başka bir söyleyişle, endüstriyel uygulamalarda robot kullanımının yaygınlaşması ile birlikte üretim kalitesi ve hassasiyeti artmıştır. Bu yaklaşımla, robot manipülatörlerinin zamanla insan eli yapısına olan benzerliklerinin artması gözlemlenmektedir. Bu çalışmada, eldivene monte edilmiş esneklik sensörleri yardımıyla insan elinden alınan konum bilgileri ile mekatronik tabanlı robotik elinin kontrolü sağlanmıștır. İnsan el parmaklarının açısal hareketleri algılanarak bir mikrodenetleyici tarafından işlendikten sonra servo motorlar yardımıyla robot elin kontrolü gerçeklenmiştir. Çalışma sonunda ortaya çıkan robot el ile insan için tehlikeli ortamlarda manipülasyon işlemlerini uzaktan kontrol etmek amaçlanmıştır. Diğer mevcut manipülatörlerin aksine daha az bir maliyet ile gerçekleştirilen bu çalışmanın ileride gerçekleştirilmesi planlanan yapay zeka tabanlı çalışmalara zemin oluşturması hedeflenmektedir.

Anahtar Kelimeler: Robot el, Flex sensör, Esnek algılayıcı kontrolü

\section{Robot Hand Design and Implementation Based on Flexible Sensor Controlled}

\begin{abstract}
One of the inputs that has an important value in the development of lean manufacturing systems increasing worldwide are the studies on robotics undoubtedly. The use of robot technologies in production is necessary if it is desired to reduce accident rates and manufacturing defects. In other words, the precision and quality in production increase day by day with the increasing use of robots in industrial applications. In parallel with this approach, it is observed to increase the similarity to human hand structure of the robot manipulators day after day. In this study, the control of the mechatronic-based robotic hand has been performed by the position information obtained from the human hand using flex sensors mounted on glove. First, the angular movements of the human fingers have been sensed and these signals have been processed by a microcontroller. Then the control of robot hand has been performed according to this data by using servo motors. At the end of the study, it has become possible to control remotely manipulation process in dangerous environments for human using robot hand. Unlike other existing manipulators, this study, which is carried out at a lower cost, aims to be pioneer for artificial intelligence based study that is planned to be carried out in the future.
\end{abstract}

Keywords: Robot hand, Flex sensor, Flexible sensor control

\section{GİRIŞ̧}

İnsan eli kavrama, sıkma, gevşetme, hissetme vb. işlevleri mükemmel bir düzeyde yapmaktadır ve robot manipülatörlerinin tasarımında bu özellikleriyle çok iyi bir örnek oluşturmaktadır. Hareketli birçok eklemin bir araya gelmesi ile oluşan elektro-mekanik bir sistem olan robot ellerin ilham kaynağı insan elidir. $\mathrm{Bu}$ sistemlerde hareket, mekanik yapıyla tahrik ve kontrolü sağlayan elektrikli parçalar vasıtasıyla sağlanır. Konu ile alakalı literatür incelendiğinde araştırmalar en genel manada insan elinin kinematik yapısına benzer robotik çalışmalar yönündedir.

İnsan eli birbirinden farklı kavrama ve duruş durumları için 4 pasif olmak üzere 24 serbestlik derecesine sahiptir. Kavrama işlemlerinin gerçekleşmesi için sirasıyla, eklemlere bağlı tendonlar, kaslar ve uyaran sinirler tarafından gerçekleşir. Günlük yaşantımızdaki aktivitelerimizin çok büyük bir kısmını karşılamak 
için, 5-6 farklı hareket yeterli olmaktadır. $\mathrm{Bu}$ hareketleri gerçekleştirebilmek için 5 serbestlik derecesi yeterli olmaktadır. Cobos ve arkadaşları çalışmalarında insan eli hareket kabiliyetlerinin kinematiklerini incelemiş ve bu hareketlere dair denklem takımlarını sunmuşlardır [1]. Endüstriyel tutucuların çoğu genellikle iki ya da üç parmaklıdır. Tek tip kavrama gerçekleştirilmekte, tek tahrikle kontrol edilmektedir. Bu tip sistemlerin kontrolü ve tasarımı kolay olabilmekte ama esnekliği yeterli olmamaktadir.

Lan ve arkadaşları çalışmalarında iki parmaklı bir insanın kavrama yapma becerisini tekrarlayan bir mikro tutucu sistemi SMA kullanarak gerçekleştirmişlerdir [2]. Ryew ve Choi çalışmalarında parmakların hareketlerinin 3 boyutlu olarak gerçekleştirilebilmesi için 2 serbestlik derecesine sahip bir eklem tasarımı gerçekleştirmişlerdir. Bu tasarım sayesinde parmak hareketleri insan parmak hareketlerine daha da benzemekte olduğu çalışmada anlaşılmıştır [3]. Malegam ve D'Silva ise çalışmalarında kol hareketlerini ve parmak hareketlerini bir önceki kaynakta belirtilen yönteme benzer bir yöntemle taklit etmeye çalışan bir sistem tasarlanmışlardır [4]. Hafiane ve arkadaşları el hareketlerinin algılanması için bilgisayarlı görü teknikleri kullanılmış ve elin 3 boyutlu modelini çıkararak SURF özellik çıkarma yöntemiyle el hareketlerini belirlenmişlerdir. $\mathrm{Bu}$ sayede teleoperasyon yani uzaktaki bir işlemin gerçekleştirilmesini amaçlamışlardır [5]. Kappassov ve arkadaşları çalışmalarında robot elin çalışması sırasında geri bildirim alınabilmesi için kullanılan yöntemleri detaylı olarak incelemiş ve bu yöntemler hakkında bilgiler sunmuşlardır [6]. Zhe $\mathrm{Xu}$ ve Emanuel Todorov, Washington üniversitesinde antropomorphic robotik el tasarımı çalışmasında bulunmuşlardır. Çalışmalarında insan elinin eklem yapısına uygun bir robotik el tasarımı sunmuşlardır [7]. Kiyoshi Hosino, Tsukuba Üniversitesinde yüksek hiz ve doğrulukta taklitçi robot el çalışmasında bulunmuştur. Çalışmasında yüksek hassasiyette hata payını azaltmak için PD kontrolü kullanmıştır. [8]. Abidhusain Syedı ve arkadaşları yeni nesil mikrodenetleyiciler kullanarak eğitim amaçlı bir robotik el tasarımı gerçekleştirmişlerdir [9]. Jayant Y. Hande ve arkadaşları çalışmalarında flex-sensör ve servo motorlardan oluşan antropomorphic robotik el tasarımı gerçekleştirmişlerdir. [10].

$\mathrm{Bu}$ çalışmada ilk olarak manipülatörün gerçeklemesinde kullanılacak olan donanımlar ve yapısal malzemeler kavramsal tasarıma tabi tutulmuş ve olası optimal sonuçlar değerlendirilmiştir. Değerlendirme sonucunda elde edilen bulgular neticesinde 3D yazıcı çıktıları ile elde edilen yapay robot elin esnek algılayıcı sensörler ve servo motorlar ile kontrolü sağlanmıştır. Kontrol kartı olarak Atmega 328 mikro denetleyicisine sahip Arduino Uno tercih edilmiştir.

\section{KAVRAMSAL TASARIM}

Gerçekleştirilen robot elin optimum düzeyde olması amacıyla öncelikli olarak kavramsal tasarım uygulanmıştır. Kavramsal tasarım aşamaları için öncelikli alanlar ise şu şekildedir;

- Geometri; Protez El ölçü parametreleri mümkün olduğunca insan eli ölçü parametrelerine uyarlanmalıdır.

- Enerji; Sisteme 6V - 9V aras1 voltaj ve 2,5A $3 \mathrm{~A}$ aras akımlık bir besleme verilmelidir.

- Çalışma Şekli ve Şartları; Sistem insan elinden alınacak hareket girdileri ile tamamen manuel olarak kontrol edilmelidir.

- Maliyet; Sistem olabildiğince ucuza mal edilmelidir.

- Emniyet; Sistem kullanıcıya ve çevreye zarar vermeden çalışabilmelidir.

Kavramsal tasarımda 5 alt fonksiyon belirlenmiştir. Bunlar sırasıyla; eldiven tasarımı, servo motor veri sinyali devreleri, kablolu haberleşme, sensör geri besleme sistemi, mikroişlemci programlama. Belirlenen öncelikli alanlar ve alt fonksiyonlar düşünüldüğünde oluşturulan çözüm varyantları Tablo 1'de verilmiştir.

Tablo 1. Kavramsal tasarım çözüm varyantları (Conceptual design solution variants)

\begin{tabular}{|c|c|c|c|c|c|}
\hline & Alt Fonksiyon & $\mathbf{1}$ & $\mathbf{2}$ & $\mathbf{3}$ & $\mathbf{4}$ \\
\hline $\mathbf{1}$ & Elektrik & Elektrik motoru & Step motor & Servo motor & Hidrolik motor \\
\hline $\mathbf{2}$ & Mekanik & Hidrolik pompa & Misina & Metal Tel & İntel Galileo Gen \\
\hline $\mathbf{3}$ & Kontrol & Arduino & PLC & Rasperry-pi & Sert plastik \\
\hline $\mathbf{4}$ & Malzeme & Metal & Ahşap & & \\
\hline $\mathbf{5}$ & Sensör & Flex & & & \\
\hline
\end{tabular}


Kavramsal tasarımı gerçekleştirilen robot el uygulamasına ait varyantlar yukarıda verilen bilgiler doğrultusunda aşağıdaki şekilde sıralayabiliriz;

- VARYANT 1: $1.1-2.3-3.2-4.1-5.1$

- VARYANT 2: $1.4-2.1-3.2-4.1-5.1$

- VARYANT 3: $1.3-2.3-3.3-4.2-5.1$

- VARYANT 4: $1.3-2.2-3.1-4.3-5.1$

- VARYANT 5: $1.3-2.2-3.4-4.3-5.1$

$\mathrm{Bu}$ beş varyant incelendiğinde; tüm işlevlerle uyumluluk, şartname isteklerini karşılama, prensipte gerçekleştirilebilirlik, müsaade edilebilir maliyet, emniyet şartlarını doğrudan karșılama, tasarım ve yeterli bilgi açısından 4.varyantın diğer varyantlara oranla uygun olduğu tespit edilmiş ve çözüm için bu varyant uygulanmıştır.

Kavramsal tasarım sonucunda gerçekleştirilecek mekatronik sisteme ait giriş elemanı esneklik sensörlerinden oluşan eldiven, çıkış elemanları ise servo motorlar olarak belirlenmiştir. Giriş ve çıkışlar arasında sistemin kararlı bir şekilde çalışmasını ve kontrolünü sağlamak amacı ile yeni nesil mikro denetleyici platformlarından Arduino tercih edilmiştir. $\mathrm{Bu}$ sayede geniş bir manüpülasyon kabiliyetini ekonomik açıdan uygun bir şekilde üretebilmek mümkün olmuştur. Sistemin oluşturulmasında kullanılan giriş, çıkış ve sistem elemanları blok diyagram şeklinde Şekil 1'de verilmiştir.

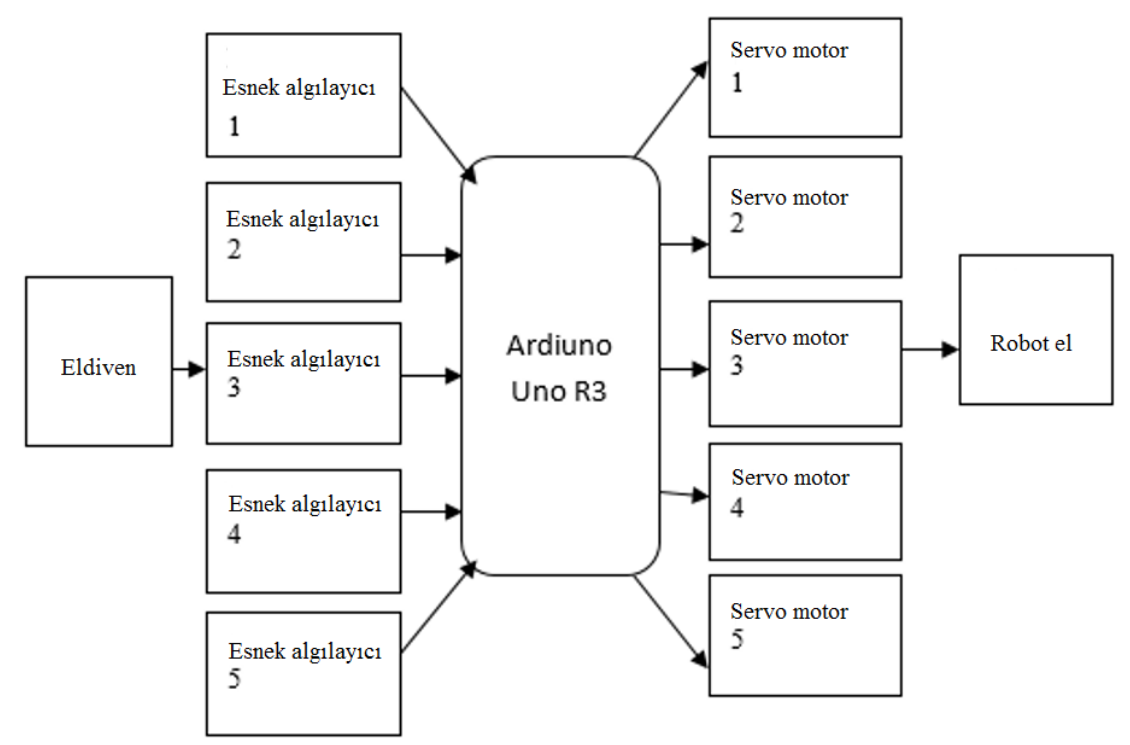

Şekil 1. Sistem elemanları blok diyagramı (System elements block diagram)

\section{KONUM ANALIZI}

İnsan el parmaklarında başparmak 2 uzuvlu ve diğer parmaklar 3 uzuvludur. Başparmak harici diğer parmaklarda işaret parmağıyla aynı uzuv sayısına sahip olduklarından konum analizleri aynıdır. Şekil 2 'de işaret parmağına ait konum analizi verilmiştir.

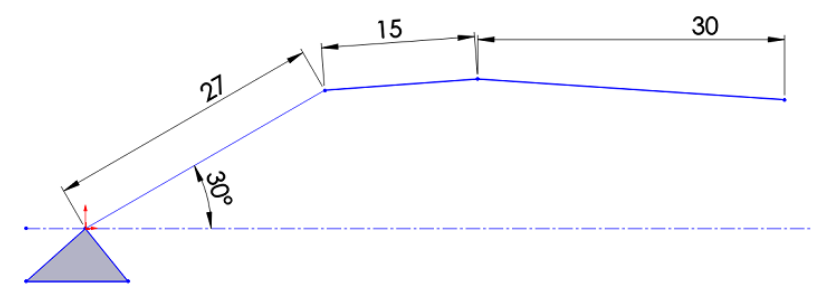

Şekil 2. İşaret parmağı konum vektörü (Index finger position vector)
$\mathrm{OA}=27 \mathrm{~mm}, \quad \mathrm{AB}=15 \mathrm{~mm}, \quad \mathrm{BC}=30 \mathrm{~mm}, \quad \mathrm{OD}=40 \mathrm{~mm}$, $\mathrm{DC}=54 \mathrm{~mm}$ olduğu kabul edilirse $\Theta_{A B}$ ve $\Theta_{B C}$ açılarının hesabı şu şekilde yapılmaktadır;

$$
\begin{aligned}
& O D+D C=O A+A B+B C \\
& 40 i+54 j=27 *(\cos 30 i+\sin 30 j)+15 *(\cos \theta a b i+ \\
& \sin \theta a b j)+30 *(\cos \theta b c i+\sin \theta b c j) \\
& 40=27 * \cos 30+15 * \cos \Theta_{A B}+30 * \cos \Theta_{B C} \\
& 54=27 * \sin 30+15 * \sin \Theta_{A B}+30 * \sin \Theta_{B C} \\
& O E=27 * \cos 30=23.4 \mathrm{~mm} \\
& A E=27 * \sin 30=13.5 \mathrm{~mm} \\
& E D=A F=O D-O E=40-23.4=16.6 \mathrm{~mm} \\
& F C=D C-D F=54-13.5=40.5 \mathrm{~mm}
\end{aligned}
$$


$A C=\sqrt{A F^{2}+F C^{2}}=\sqrt{16.6^{2}+40.5^{2}}=43.8$

Tan $\alpha=C F / A F=40.5 / 16.6 \rightarrow \alpha=67.7^{\circ}$

$B C^{2}=A B^{2}+A C^{2}-2 * A B^{*} A C^{*} \cos \beta$

$30^{2}=15^{2}+43.8^{2}-2 * 15^{*} 43.8^{*} \cos \beta \rightarrow \beta=18.9^{\circ}$

$\Theta_{A B}=\alpha+\beta=67.7+18.9 \rightarrow \Theta_{A B}=8$ 86. $^{\circ}$

$40=23.4+15 * \cos (86.6)+30 * \cos \theta_{B C}$

$\rightarrow \theta_{B C}=58.4^{\circ}$

\section{TASARIM}

Projede protez elin katı model çizimleri CAD programında çizilmiştir. Çizilen parçalar .stl uzantısına dönüştürülerek 3D yazıcıda basıma hazırlanmıştır. Protez elin teknik resim detayları için katı modelleme sistemlerinden yararlanılmıştır. Şekil 3'te tasarım aşamasında oluşturulan parçalara ait görsellere yer verilmiştir.

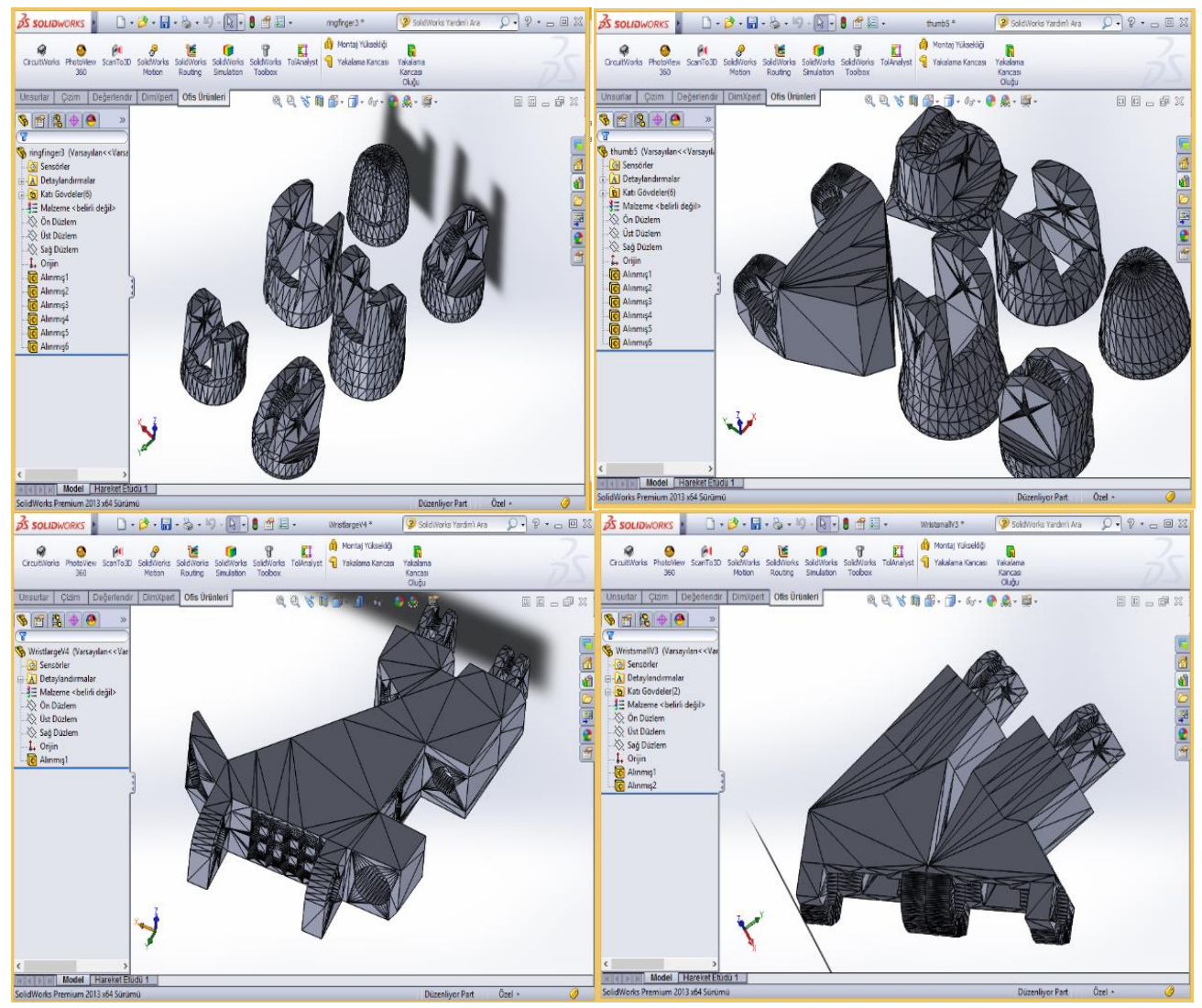

Şekil 3. Robot el parçalarının tasarımları (Parts of robot hand designs)

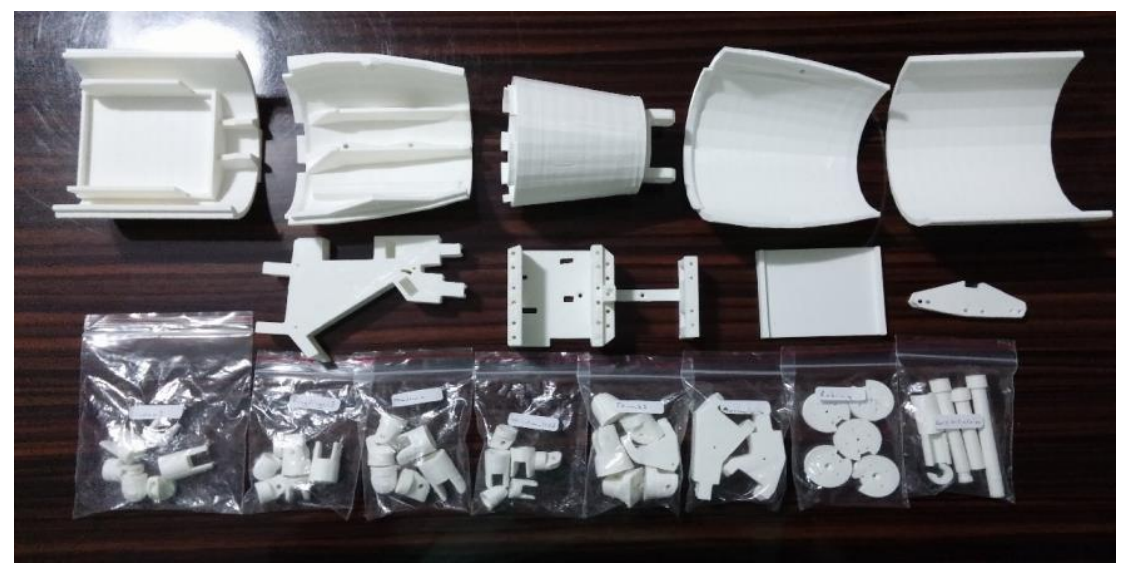

Şekil 4. 3D yazıcı parça çıktıları (3D printer parts output) 
Tasarımı gerçekleştirilen parçaların 3D yazıcı ile çıktıları alınmış ve montaja hazır hale getirilmiştir. ŞeQil 4'te gösterilen şekliyle baskısı alınan parçaların montajinda belirlenen bölgelere servo motorlar konulmuștur. alınarak tasarımı yapılan robot ele ait 3D yazıcı çıktı parçalarının montajlanmış hali Şekil 5'te verilmiştir. Çalışmada sonucu oluşturulan bu robot el Ayrıca PLA malzemeden üretilmiş olması da robot elin taşınabilirliği açısından önem arz etmektedir.

Mümkün olduğunca ergonomik hareket dikkate

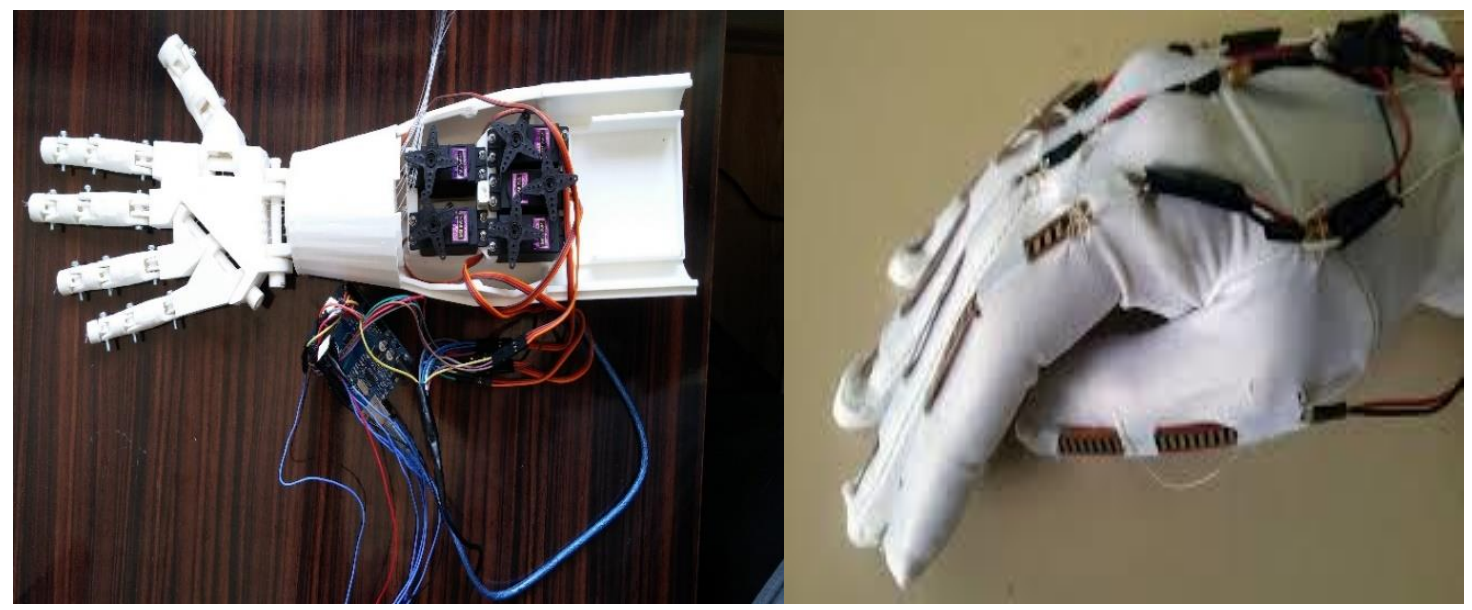

Şekil 5. Montajı tamamlanan robot el (Assembled robot hand)

\section{SONUÇ VE ÖNERILER}

Yapılan testlerde esneklik algılayıcı sensörlerin, insan elinin hareketlerini uygun bir şekilde ölçebildiği görülmüştür. Robot el ile insan eli parmak açılarında $\% 5$ oranında açısal farklılıklar gözlemlenmiștir. Bu farkların en önemli nedeni esneklik sensörlerdeki direnç değişiminin voltaj bölücü prensibiyle okunmasıdır. $\mathrm{Bu}$ bakımdan her parmağın eklem yerlerinde bağımsız sensörler kullanılarak sistemin performansı artırılabilir. Bir sonraki aşamada; Robotik elin kontrolünü kablosuz hale getirerek sadece parmak değil insan kolunun bütün hareketlerini taklit edebilecek bir sistem tasarımı gerçeklenmesi hedeflenmektedir.

\section{KAYNAKÇA}

[1] Salvador Cobos, Manuel Ferre, M.A. Sanchez Uran, Javier Ortego and Cesar Pena, "Efficient Human Hand Kinematics for Manipulation Tasks", 2008 IEEE/RSJ International Conference on Intelligent Robots and Systems Acropolis Convention Center, Nice, France, 2008

[2] Chao-Chieh Lan, Che-Min Lin, Chen-Hsien Fan, "A Self-Sensing Microgripper Module With Wide Handling Ranges,", IEEE/ASME Transactions on Mechatronics, vol.16, no.1, pp.141-50,
doi:10.1109/TMECH.2009.2037495, 2011

[3] Ryew, S., Hyoukryeol Choi, "Doubleactive universal joint (DAUJ): robotic jointmechanism for human-like motions," in,IEEE Transactions on Robotics and Automation, vol.17, no.3, pp.290-300, doi: 10.1109/70.938386, 2001

[4] Malegam, K.D.; D'Silva, M.S.,"Mimicking robotic hand-arm," 2011 Annual IEEE India Conference (INDICON ), pp.1-5, 16-18, doi: 10.1109/INDCON.2011.6139365, 2011

[5] Hafiane, S., Salih, Y., Malik, A.S., "3Dhand recognition for telerobotics," 2013IEEE Symposium on Computers \&Informatics (ISCI), pp.132-137, doi: 10.1109/ISCI.2013.6612390, 2013

[6] Zhanat Kappassov, Juan-AntonioCorrales, Véronique Perdereau, "Tactile sensing in dexterous robot hands - Review",Robotics and Autonomous Systems, Volume 74, Part A, Pages195-220, ISSN 0921-8890, doi: dx.10.1016/j.robot.2015.07.015, 2015 [7] Zhe Xu and Emanuel Todorov, "Design of a Highly Biomimetic Anthropomorphic Robotic Hand towards Artificial Limb Regeneration", Robotics and Automation (ICRA), 2016 IEEE International Conference on, DOI: 10.1109/ICRA.2016.7487528, 2016

[8] Kiyoshi Hoshino, "Copycat Hand - Robot Hand Generating Imitative Behaviour at High Speed and with High Accuracy", Humanoid Robots, New 
Developments, Book edited by: Armando Carlos de Pina Filho ISBN 978-3-902613-02-8, pp.582, I-Tech, Vienna, Austria, 2007

[9] Abidhusain Syed, Zamrrud Taj H. Agasbal, Thimmannagouday Melligeri, Bheemesh Gudur, "Flex Sensor Based Robotic Arm Controller Using Micro Controller", Journal of Software Engineering and Applications, 364-366, http://dx.doi.org/10.4236/jsea.2012.55042, 2012
[10] Jayant Y. Hande, Niket Malusare, SubodhSawarbandhe, HarshalDarbhe, "Design for Robotic Hand Using Flex-sensor", International Journal of Advanced Research in Electronics and Communication Engineering (IJARECE), page 28462850, Volume 4, Issue 12, 2015 